\title{
The association of the uterine motion with bladder volume during radiotherapy in gynecological malignancies
}

\author{
Bhandari Virendra, Mutneja Abhinav, Gurjar Omprakash, Saadvik Raghuram, Bagdare Priyusha, \\ Gupta Krishnlal, Singh Kanchan \\ Department of Radiation Oncology, Sri Aurobindo Medical College and PG Institute, Indore 452001, India.
}

Correspondence to: Dr. Bhandari Virendra, Department of Radiation Oncology, Sri Aurobindo Medical College and PG Institute, 401, Samyak Towers, 16/3 Old Palasia, Indore 452001, India. E-mail: virencancer@yahoo.co.in

\section{A B S T R A C T}

Aim: This study was performed to assess the extent of interfraction uterine motion during radiotherapy for cervical cancer and uterine body carcinoma while maintaining a strict bladder filling protocol. Methods: Twenty-four patients with cervical cancer or uterine body carcinoma who were treated on a linear accelerator, were recruited. During the course of external beam radiotherapy, cone beam computed tomographic scans were taken, once at the start of treatment and then weekly until the completion of the radiotherapy course. Patients were instructed to maintain a strict bladder filling protocol. After negating the effect of patient's setup error by offline cone beam computed tomographic imaging, the position of the uterus was defined in the clinical target volume. Then the position of the uterus was compared in the following weekly scans. The position of the uterus was also correlated with the position and the filling of the bladder. This change in uterus position was measured separately in the anterioposterior (AP), superioinferior (SI), and lateral directions. Results: According to calculations based on weekly imaging, The mean values of shift in AP, SI, and lateral directions were respectively $0.67,0.29$, and 0.23 The mean extent of motion in the uterine position on a daily basis for individual patients ranged from -2.28 to +1.3 in AP, -0.56 to +0.71 in SI, and from -0.6 to +0.45 in lateral directions. Conclusion: At least once a week cone beam computed tomography might be necessary to minimize the geometrical miss and deliver the planned doses to the target tissue and normal structure provide best results with minimum toxicity by maintaining a bladder volume of about $100 \mathrm{~mL}$ and an empty rectum during the whole course of treatment. The daily anatomical shift and contour of the patients maintaining a bladder volume of approximately $100 \mathrm{~mL}$ with an empty rectum may result in asymmetrical conforming to the planning target volume and hence appropriate and adequate planning target volume margins are required.

Key words: Uterine motion; weekly computed tomographic evaluation; intensity modulated radiotherapy; inter-fraction variation; bladder volume

\section{INTRODUCTION}

External beam radiotherapy (EBRT) plays a great role in the management of female gynecologic cancers. Intensity modulated radiotherapy (IMRT) and image guided radiotherapy (IGRT) are considered the treatment of choice for cervical cancer and uterus carcinomas. These new techniques have overtaken the conventional four-field box technique as the preferred modality of treatment and have proven more efficacious in various studies. ${ }^{[1-3]}$ The IGRT further reduces the radiation dose to the organs at risk (OAR) and thus further reduces toxicities. On the other hand, IMRT has very strict clinical and planning target volumes (CTV and PTV respectively) conforming to a particular volume to

\begin{tabular}{|l|l|}
\hline \multicolumn{2}{|c|}{ Access this article online } \\
\hline Quick Response Code: & Website: \\
\hline & http://jcmtjournal.com \\
\cline { 2 - 3 } & \\
\hline
\end{tabular}

spare the adjacent organs; however, it does not account for interfraction and intrafraction motion of various organs as well as reduction in tumor volume during treatment.

This phenomenon did not need to be considered in the conventional technique as it provided a uniform dose to all the structures included in the treatment field which avoids a geometrical miss of the tumor. Therefore, motion of organs within the treatment area is a vital issue in IMRT and three dimensional conformal radiotherapy (3D-CRT). During the treatmenta steep dose gradient is usually present that uses

This is an open access article distributed under the terms of the Creative Commons Attribution-NonCommercial-ShareAlike 3.0 License, which allows others to remix, tweak, and build upon the work non-commercially, as long as the author is credited and the new creations are licensed under the identical terms.

For reprints contact: service@oaepublish.com

How to cite this article: Virendra $B$, Abhinav M, Omprakash $G$, Raghuram S, Priyusha B, Krishnlal G, Kanchan S. The association of the uterine motion with bladder volume during radiotherapy in gynecological malignancies. J Cancer Metasta Treat 2016;2:139-43.

Received: 03-09-2015; Accepted: 19-12-2015. 
the newer techniques that conform to the optimum dose of the CTV or the shape of the target volume. Even a little geometrical movement could result in an underdosing to the target volume or conversely, delivering high undesirable doses to the surrounding normal tissues. These effects highlight the importance of accurate margin determination.

This pilot study was conducted to define the daily uterine shift in patients undergoing external radiotherapy on linear accelerators with IMRT technique using IGRT with the help of an on-board cone beam computed tomography (CBCT) scan taken once a week during the whole course of radiotherapy.

\section{METHODS}

We recruited 24 patients with the ages of 45 and 70 years who were diagnosed with cervical cancer and uterine body carcinoma were treated with EBRT (50 Gy in 25 fractions) from September 2010 to December 2013, and opted for the IMRT technique.

Before starting radiotherapy a six-clamp thermoplastic Orfit cast was prepared for immobilization of the pelvic region in all the patients and then contrast enhanced computed tomographic (CT) scan of pelvis was done and 3 $\mathrm{mm}$ slice thickness scans were acquired and transferred to the treatment planning system (TPS) (Eclipse version 8.9). The gross tumour volume (GTV), CTV, PTV, and organs at risk (OAR) such as rectum, bladder, and femoral heads were delineated on the $\mathrm{CT}$ images following the guidelines of the International Commission on Radiation Units and Measurements report number 83 (ICRU 83). ${ }^{[4]}$ Then IMRT plans were created with 6 Mega Volt (MV) and $15 \mathrm{MV}$ photon beam and a Varian leaf motion calculator (version 8.9.08), was utilized to calculate leaf motion for dynamic dose delivery. Dose-volume optimizer was used for plan optimization. Anisotropic analytical algorithm was used to calculate doses with grid size of $0.25 \mathrm{~cm}$. After approving, the plans were scheduled for 25 fractions with daily imaging by On Board Imaging system and CBCT technique.

Patients were positioned and immobilized with the orfit cast on couch and then CBCT was done with the OBI system. The anatomy matching software Portal Vision 7.5, was used to study the patient's setup deviations and to determine the spatial coordinates in the images. After patient setup and laser alignment during EBRT, a kV portal image was taken and matched with the reference image to avoid patient's setup error and a CBCT scan was performed once at the start of treatment and then weekly until the completion of treatment. This CBCT was matched with the reference CT image to see the shift of the uterus, which was noted in $\mathrm{X}, \mathrm{Y}$, and $\mathrm{Z}$ axes [Figures 1 and 2]. A total of 96 scans were obtained during the whole treatment period, ie, four scans for each patient. Then the patients were assessed for intracavitory brachytherapy and if they did not fit, they

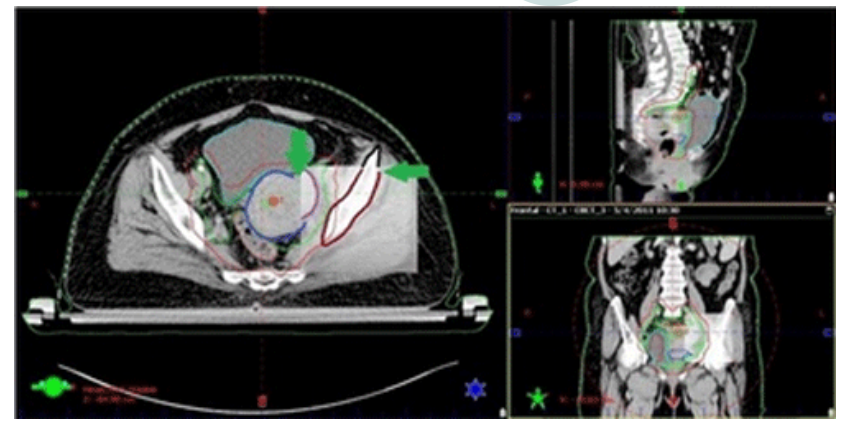

Figure 1: The perfect bone to bone matching of a patient with the reference computed tomographic image and the uterine shift between the two scans to negate the effect of the patient's setup errors

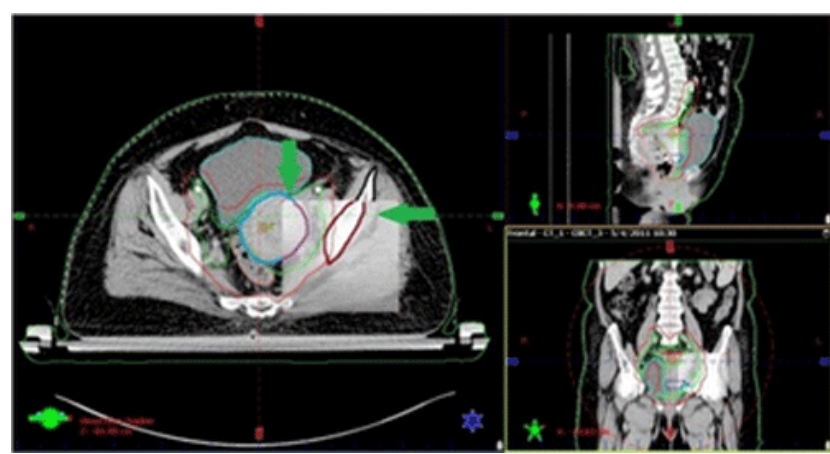

Figure 2: The soft tissue matching of the contoured uterus with the reference scan showing bone displacement after radiotherapy

were continued for boost by EBRT.

Patients were asked to maintain a strict bowel and bladder filling protocol by instructing all the patients to defecate and urinate and then to maintain strict water intake of around $200 \mathrm{~mL}$ of water $20 \mathrm{~min}$ before the procedure. The position of the uterus was defined in the CTV during delineation on axial images of the lesion for radical radiotherapy. The CTV included all the gross as well microscopic lesions. The OARs such as the bladder, rectum, intestines, andfemoral heads were also delineated on axial images.

The position of the uterus was then compared in the following weekly scans on the axial images guided by sagittal, coronal and three-dimensional reconstructions. This was done by merging the weekly CT images with the reference $\mathrm{CT}$ image taken before the start of the treatment at the same level. For every scan, we used the lower level of the S1 vertebra. After merging the images, a preliminary bone to bone matching was done to negate the effect of patient's setup errors which was followed by soft tissue matching of the uterus in two CT images. The change in CTV position during the bone to bone matching was subtracted from the anteroposterior (AP), superoinferior (SI), and lateral changes during the soft tissue matching. The correlation between the position of uterus with the position and the filling of the bladder was also assessed. This change in uterus position was measured separately in the AP, SI and lateral directions. No additional effort on the part of the patient or the doctor was required because a part of the OBI software performed the measurements during 
the treatment. The time taken for every treatment was also similar among the patients undergoing IGRT of the pelvic region. The Mean of all the obtained-values for each patient was calculated and an unpaired-one-sample student $t$-test was applied to obtain the significance. The $P$ value is less than 0.001 which is highly significant.

\section{RESULTS}

The mean, standard deviation, and median of uterine motion in each plane were calculated to see its association with the bladder filling and its influence on the displacement of the uterus. As shown in the Table 1, the displacement ranges were significant depending on the patient, although the mean values of the displacement were within $1 \mathrm{~cm}$. The mean values of shift in AP, SI, and lateral directions were respectively $0.67,0.29$, and 0.23 for all the 96 scans done for 24 patients over the period of EBRT [Table 2].

The mean extent of motion in the uterine position on a daily basis for individual patients ranged from -2.28 to +1.3 in AP, -0.56 to +0.71 in SI, and from -0.6 to +0.45 in lateral directions. The mean movement in all the directions was also calculated over the course of the full treatment [Figure 3], and showed more anterior and superior shift that might be due to bladder filling while the lateral deviation, although

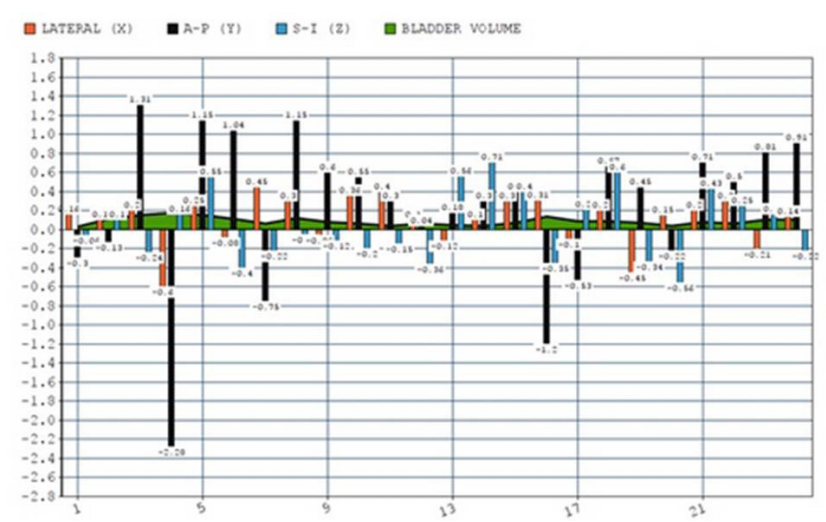

Figure 3: The mean change of the uterine position in the lateral $(X)$ anteroposterior $(\mathrm{Y})$, and the superoinferior $(\mathrm{Z})$ directions per patient over the whole course of the treatment along with its association with the bladder volume depicted in the area curve

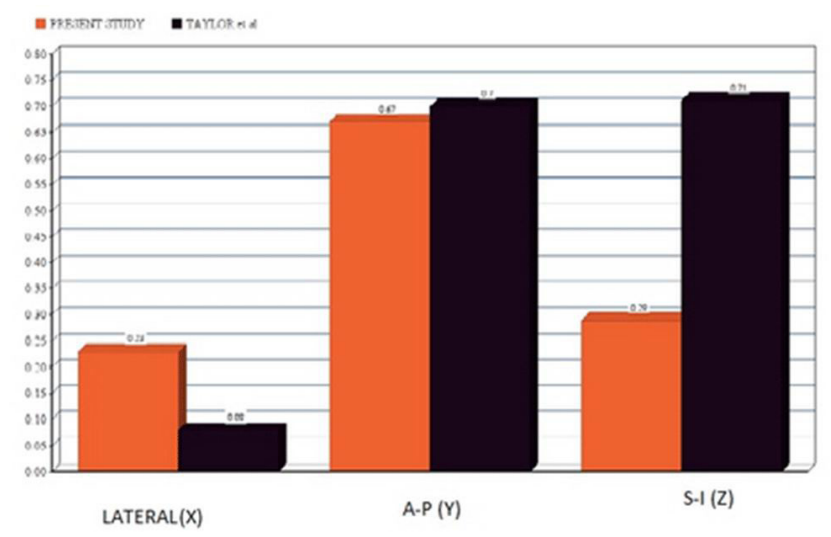

Figure 4: Comparison of different means of displacement in different directions between current study and the study of Taylor et al. ${ }^{[11]}$
Table 1: Combined uterine motion in three different dimensions in patients undergoing radiotherapy

\begin{tabular}{lccc}
\hline Dimensions & $\begin{array}{c}\text { Mean (SD), } \\
\text { cm }\end{array}$ & $\begin{array}{c}\text { Median, } \\
\text { cm }\end{array}$ & $\begin{array}{c}\text { Range of } \\
\text { motion, cm }\end{array}$ \\
\hline Lateral $(\mathrm{X})$ & $0.23(0.22)$ & 0.2 & -0.6 to 0.45 \\
Anteroposterior (Y) & $0.67(0.83)$ & 0.57 & -2.28 to 1.3 \\
Superoinferior (Z) & $0.29(0.40)$ & 0.245 & -0.36 to 0.71 \\
\hline
\end{tabular}

present, was minimum. The posterior shift might be due to the rectal filling or presence or absence of gas in the rectum.

The mean bladder volume was calculated to be $90.55 \mathrm{~mL}$ for all patients, and each patient had an average bladder volume of about $80 \mathrm{~mL}$ to $100 \mathrm{~mL}$ over the course of their treatment. This was done by maintaining a strict bladder control protocol for each patient. We found that maximum range of motion was observed when the bladder volume exceeded $100 \mathrm{~mL}$ as was seen in patient number 4, where a mean maximum shift in AP direction was almost up to $-2.8 \mathrm{~cm}$. When it was compared with their mean bladder volume, it was found to be excessive with a mean of almost up to $180 \mathrm{~mL}$ during the course of their treatment.

\section{DISCUSSION}

The EBRT with radiation doses of 40 to 50 Gy followed by boost with brachytherapy has been proven to be effective in the local control of cervical and uterine cancers. However, one of the major concerns with this modality of treatment has been acute or chronic small bowel toxicities with advancement in the treatment techniques of radiation therapy, it has been possible to reduce the toxicity to bowel. Nonetheless, it is essential to see that the benefits are not achieved at the cost of decreased local control due to a geometrical miss. ${ }^{[5-7]}$

The CTV for primary cervical cancer treatment comprises the partially mobile uterus and cervix, the less mobile upper vagina, parametrium and pelvic lymph nodes located along the side walls in the pelvis. When treating with a conventional four field box technique, internal motion is less critical as the dose distribution is likely to encompass the central structures within the high dose region even if they move a little. The dose distribution in IMRT has the potential to conform more precisely to the target volume. Therefore, assessment of organ motion has become more important as there can be a geometrical miss during daily treatment.

According to the ICRU statement number 62 (ICRU 62) two margin volumes of CTV should be used to create the PTV: the internal margin to account for organ motion, and the setup marginto account for variation in patient position. ${ }^{[8]}$ Huh et al. ${ }^{[9]}$ and Lee et al. ${ }^{[10]}$ have previously reported the changes in the uterus position by comparing two magnetic resonance images taken before and during the period of radiotherapy. They showed that uterus movement and its 
Table 2: The mean of movement in all directions and the mean bladder volume for each patient

\begin{tabular}{lcccc}
\hline \multirow{2}{*}{ Patient No. } & \multicolumn{3}{c}{ Dimensions } & \multirow{2}{*}{ Bladder volume, L } \\
\cline { 2 - 4 } & Lateral $(\mathbf{X}), \mathbf{c m}$ & Anteroposterior (Y), cm & Superoinferior (Z), cm & 0.031 \\
1 & 0.16 & -0.3 & 0.06 & 0.111 \\
2 & 0.1 & -0.133 & -0.24 & 0.151 \\
3 & 0.2 & 1.32 & 0.16 & 0.189 \\
4 & -0.6 & -2.28 & 0.55 & 0.157 \\
5 & 0.25 & 1.15 & -0.4 & 0.113 \\
6 & -0.08 & 1.04 & -0.225 & 0.062 \\
7 & 0.45 & -0.75 & -0.05 & 0.125 \\
8 & 0.3 & 1.15 & -0.12 & 0.088 \\
9 & -0.06 & 0.6 & -0.2 & 0.067 \\
10 & 0.36 & 0.55 & -0.15 & 0.046 \\
11 & 0.4 & 0.3 & -0.36 & 0.064 \\
12 & 0.1 & 0.04 & 0.56 & 0.055 \\
13 & -0.12 & 0.18 & 0.71 & 0.049 \\
14 & 0.1 & 0.3 & 0.4 & 0.076 \\
15 & 0.3 & 0.4 & -0.35 & 0.140 \\
16 & 0.31 & -1.21 & 0.21 & 0.096 \\
17 & -0.1 & -0.53 & 0.6 & 0.090 \\
18 & 0.2 & 0.67 & -0.34 & 0.070 \\
19 & -0.45 & 0.45 & -0.56 & 0.040 \\
20 & 0.15 & -0.22 & 0.43 & 0.083 \\
22 & 0.2 & 0.71 & 0.25 & 0.066 \\
23 & 0.3 & 0.51 & 0.16 & 0.100 \\
24 & -0.21 & 0.81 & -0.22 & 0.105 \\
\hline
\end{tabular}

Table 3: The comparison of the magnitude of displacements between current study and the study of Taylor et al. ${ }^{[1]}$

\begin{tabular}{lccc}
\hline \multicolumn{4}{c}{ Magnitude of displacement, $\mathbf{c m}$} \\
\hline Dimensions & Mean (SD) & Median & Range \\
\hline Present study & & & \\
Lateral & $0.23(0.22)$ & 0.2 & -0.6 to 0.45 \\
Anteroposterior & $0.67(0.83)$ & 0.57 & -2.28 to 1.3 \\
Superoinferior & $0.29(0.40)$ & 0.24 & -0.36 to 0.71 \\
Taylor et al. ${ }^{[1]}$ study & & & \\
Lateral & $0.08(0.13)$ & 0.0 & 0 to 0.5 \\
Anteroposterior & $0.7(0.9)$ & 0.5 & 0 to 0.48 \\
Superoinferior & $0.71(0.68)$ & 0.5 & 0 to 0.32 \\
\hline
\end{tabular}

An assessment of interfractional uterine and cervical motion: implications for radiotherapy target volume definition in gynaecological cancer

Table 4: Adverse effects of radiotherapy in twenty-four participants

\begin{tabular}{lcc}
\hline & \multicolumn{2}{c}{ Frequency of toxicity, \% } \\
\cline { 2 - 3 } & Grade 1 & Grade 2 \\
\hline Dysuria & 80 & 20 \\
Urinary frequency/urgency & 90 & 10 \\
Diarrhea & 95 & 5 \\
\hline
\end{tabular}

positional change were significant, which suggested the importance of accurately determining the target mobility for the conformal treatment. However, in that study, two sets of magnetic resonance images were taken in the supine position without a small bowel displacement system (SBDS) while the patients were treated in prone position with a SBDS placed under the patient's abdomen. We studied the uterine motion once a week during the full course of radiotherapy and every time, we found a significant shift in uterine position in all directions.

In our study the mean bladder volume was $90.55 \mathrm{~mL}$ and it was shown that major shift occurred if the bladder volume exceeded $100 \mathrm{~mL}$. We expected a mean bladder volume of 80 to $120 \mathrm{~mL}$ during IMRT in all our patients and this was corroborated on the weekly CBCT scans. Despite maintaining a standard bladder volume, we saw a uterine shift daily.

In a similar study by Taylor et al.,${ }^{[1]}$ in addition to the uterus, the movement of the cervix was assessed to determine the internal margin for radiotherapy. They concluded that an asymmetrical margin with CTV-PTV expansion of the uterus and cervix was needed during the treatment while they emphasized on the need for a strict bladder and rectum filling protocol during treatment. ${ }^{[11]}$ Very few studies have documented the changes in uterine position during the radiotherapy course. An association between bladder filling and uterine movement was reported by Buchali et al. ${ }^{[12]}$ that indicated no major AP change in cervical position. A maximum mean displacement in AP direction was seen in our study. In contrast, the mean displacement in SI direction was also substantial in the study by Taylor et al. ${ }^{[11]}$ [Table 3 and Figure 4]. The difference might be due to strict adherence to the bladder-bowel filling protocol in our study as well as once weekly CBCT instead of two imaging on two consecutive days in their study.

There is a definite association between the bladder filling and rectum emptying with uterine cervix movement. In addition, this association has been demonstrated by studies that have assessed the association of the bladder and rectum volume with the displacements of the uterus and the cervix respectively. Moreover, maximum uterine motion at the fundus emphasizes on the need to contribute a variable 
margin around the fundus in the uterine shift.

The incidence of early bladder and rectal toxicities amongst all our patients were mild with all of them except two showing Grade 2 cystitis and mild diarrhea. Only one patient had Grade 2 diarrhea which was controlled with conservative measures [Table 4].

Despite maintaining a strict bladder filling and rectal emptying protocol, the interfraction movement in the uterine position during the course of radiotherapy may lead to a miss in target or overtreatment of the rectum, which lead to toxicity. We could not find any study in which uterine shift was observed during the whole course of radiotherapy hence, we were unable to compare the results with other works.

In conclusion, interfraction movement of the target organs may lead to overdosing or underdosing of the target or the normal structures during IMRT, hence at least once a week CBCT imaging might be necessary to minimize the geometrical miss of the tumor and deliver the planned doses to the target and normal structures for the best local control with minimum toxicity which is the primary aim of IMRT. This would also aid in the selection of appropriate and adequate planning target margins and provide an asymmetrical PTV conforming to the daily anatomical shift and contour of the patients. We also recommend a tapered CTV to PTV margin especially around the fundus of the uterus as maximum uterine motion is known at the fundus however further studies with larger numbers of patients and exact point localization of the uterus will be required for this purpose.

\section{Financial support and sponsorship}

Nil.

\section{Conflicts of interest}

There are no conflicts of interest.

\section{REFERENCES}

1. Mundt AJ, Lujan AE, Rotmensch J, Waggoner SE, Yamada SD, Fleming G, Roeske JC. Intensity-modulated whole pelvic radiotherapy in women with gynecologic malignancies. Int J Radiat Oncol Biol Phys 2002;52:1330-7.

2. Portelance L, Chao KS, Grigsby PW, Bennet H, Low D. Intensity modulated radiation therapy (IMRT) reduces small bowel, rectum, and bladder doses in patients with cervical cancer receiving pelvic and paraaortic irradiation. Int J Radiat Oncol Biol Phys 2001;51:261-6.

3. Roeske JC, Lujan A, Rotmensch J, Waggoner SE, Yamada D, Mundt AJ. Intensity-modulated whole pelvic radiation therapy in patients with gynecologic malignancies. Int $J$ Radiat Oncol Biol Phys 2000;48:1613-21.

4. ICRU Report 83. Prescribing, Recording, and Reporting Photon-Beam Intensity-Modulated Radiation Therapy (IMRT). Bethesda, MD: International Commission on Radiation Units and Measurements; 2010.

5. Gerstner N, Wachter S, Knocke TH, Fellner C, Wambersie A, Pötter $\mathrm{R}$. The benefit of Beam's eye view based 3D treatment planning for cervical cancer. Radiother Oncol 1999;51:71-8.

6. Weiss E, Eberlein K, Pradier O, Schmidberger H, Hess CF. The impact of patient positioning on the adequate coverage of the uterus in the primary irradiation of cervical carcinoma: a prospective analysis using magnetic resonance imaging. Radiother Oncol 2002;63:83-7.

7. Thomas L, Chacon B, Kind M, Lasbareilles O, Muyldermans P, Chemin A, Le Treut A, Pigneux J, Kantor G. Magnetic resonance imaging in the treatment planning of radiation therapy in carcinoma of the cervix treated with the four-field pelvic technique. Int J Radiat Oncol Biol Phys 1997;37:827-32.

8. ICRU Report 62. Prescribing, recording and reporting photon beam therapy (supplement to ICRU report 50). Bethesda, MD: International Commission on Radiation Units and Measurements; 1999.

9. Huh SJ, Park W, Han Y. Interfractional variation in position of the uterus during radical radiotherapy for cervical cancer. Radiother Oncol 2004;71:73-9.

10. Lee JE, Han Y, Huh SJ, Park W, Kang MG, Ahn YC, Lim do H. Interfractional variation of uterine position during radical RT: weekly CT evaluation. Gynecol Oncol 2007;104:145-51.

11. Taylor A, Powell ME. An assessment of interfractional uterine and cervical motion: implications for radiotherapy target volume definition in gynaecological cancer. Radiother Oncol 2008;88:250-7.

12. Buchali A, Koswig S, Dinges S, Rosenthal P, Salk J, Lackner G, Böhmer D, Schlenger L, Budach V. Impact of the filling status of the bladder and rectum on their integral dose distribution and the movement of the uterus in the treatment planning of gynaecological cancer. Radiother Oncol 1999;52:29-34. 